\title{
ACCESSING REMOTE ANDROID MOBILE CONTENTS
}

\author{
S. S. Damawale ${ }^{1}$ \\ ${ }^{1}$ Assistant Professor, CSE Department, N.K.Orchid College of Engineering \& Technology, Solapur, Maharashtra, \\ India. \\ Sujata.damawale11@gmail.com
}

\begin{abstract}
Now a day's everyone is using a mobile phone and it is not only used for calling purpose. Today all mobile Users are using android phone and can store images, videos, files, contacts etc. Mobile phones now a day are not only for calling purpose but also for important work like banking, corporate, education etc. In case, if user forgets mobile at home and wants some important data from it remotely from one place to another place. For this there are several android applications available for smart phones today that can help one to get it. This paper is presenting an android app for the same "Accessing Remote Android Mobile Contents". This app will take user requirement as input and retrieve it. This application requires internet connection first, if it is connected it will open login prompt. In which user has to provide unique user id and password which is decided during installation of app. Once the user is validated by remote mobile phone, user will see a menu on his/her screen. User has to select menu option form that and send it to remote phone. User will receive data (contacts) from remote mobile.
\end{abstract}

Keywords: Android, Remote Mobile, Local Mobile, Eclipse.

\section{I . INTRODUCTION}

In today's faster life mobile phone essential part of human life. Mobile phones now a day are not only used by corporate peoples, but also it is useful for students, doctors, service mans, engineers for their day to day activities.

The use of mobile phone now a days is not limited up speaking only, it is used for many purposes like recording, video making, transmitting information, connecting phone to computer. Mobile phone is playing an vital role.

Suppose you forgot your phone at home and you want some data from your mobile phone urgently like contacts, text documents. In such cases you would need some application which will provide facility to access the contents of mobile remotely. Today there is an application for every other things so why not to have an application which will let you access the contents of mobile phone remotely.

"Accessing Remote Android Mobile Contents" is an application that works on android based smart phones. To use this application the user should be authenticated. After authentication he/she has to select option from list depending upon which type of information he/she wants.

\section{EXISTING SYSTEM}

The phoneaway application which is used by users to access their own mobile phone contents remotely. To use phoneaway application the application should be installed in user's own mobile phone. The phoneaway is available on google freely.
When user install the phoneaway application in own mobile, user have to register another mobile number. That number is useful to get all the information from users own mobile.

The use of phoneaway application is used to find and search contacts which are in users own mobile. User can locate their mobile phone when it lost.

After installing, phoneaway application runs in the background in user's own mobile phone. After installing the application in users Smartphone User have to register an another mobile number in the application, that mobile number is used to access information from users own mobile phone, and only one alternative number can be registered.

\section{METHODOLOGY}

This section describes the system architecture. Figure1 shows the design architecture, which shows the flow of application.

First part is user interface; the user interface shows the first part. i.e graphical user interface where user can interact. There are different components to show the interface, which include authentication, connectivity etc.

The local mobile indicates the phone on user side (any random phone) which user can operate, that user can choose to do the connectivity to remote phone. Using $3 \mathrm{G}$ internet service the local mobile can connect with remote mobile anywhere. Both the androids mobiles should be register with this application to use the service. Suppose remote mobile is at home and you are at another location. 


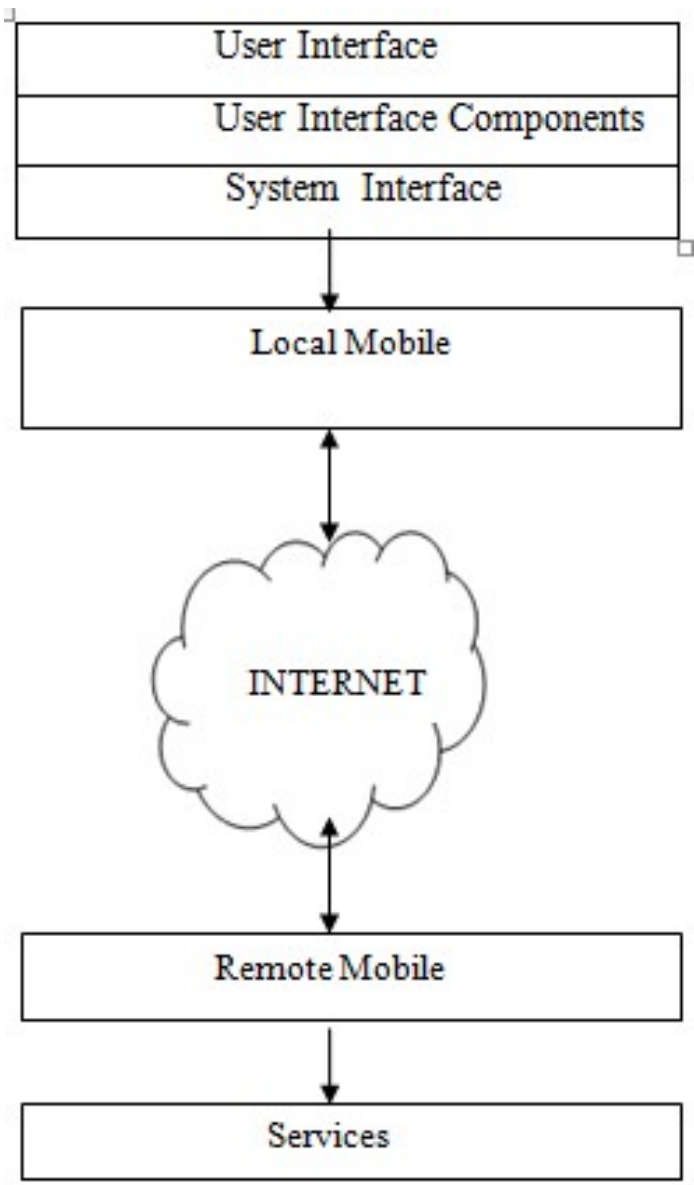

Fig.1. System Architecture

If you want some data urgently which is in your android mobile then this application will help you. Here the mobile number as username and password for authentication and IP address to establish connection between two mobiles. The user has to give the same mobile number and password as he had given during installation of application. After filing all the required inputs now user has to click on the "Login" button in order to traverse to the next screen. When the user click on login button The IP address of that mobile automatically binds to the edit text of Ip Address and one specific format of SMS will be sent to the mobile which is at home which will cost 1 Rs. After receiving the SMS, the application automatically starts on the mobile which is at home i.e. remote mobile. Then it tokenize the SMS and authenticate the SMS contents with the file contents which we have taken during installation. When authentication is successful it automatically turn on the data connection if it is off and connection is established using IP address in SMS. Once the connection is established it sends a message as "CONNECTED" to the local mobile. after receiving the message "CONNECTED". Here user has to select either CONTACTS or FILES. When user selects on CONTACTS. It displays a screen where user have to enter something to retrieve from remote mobile. Here user have to give the name of the person whose number he wants. After clicking on "send" button this name is send to the remote mobile which is at home. After few seconds, name with contact number is display in the list view.

\section{DETAILS OF PROPOSED SYSTEM}

This section describes all detail system of the application, all designing of the application. Following are the different designing diagrams of the application.

\section{A. Data Flow Diagrams}

Data flow diagram means which takes input, process that input and represent output. Data flow diagram is view of system i.e. how data flow in system.

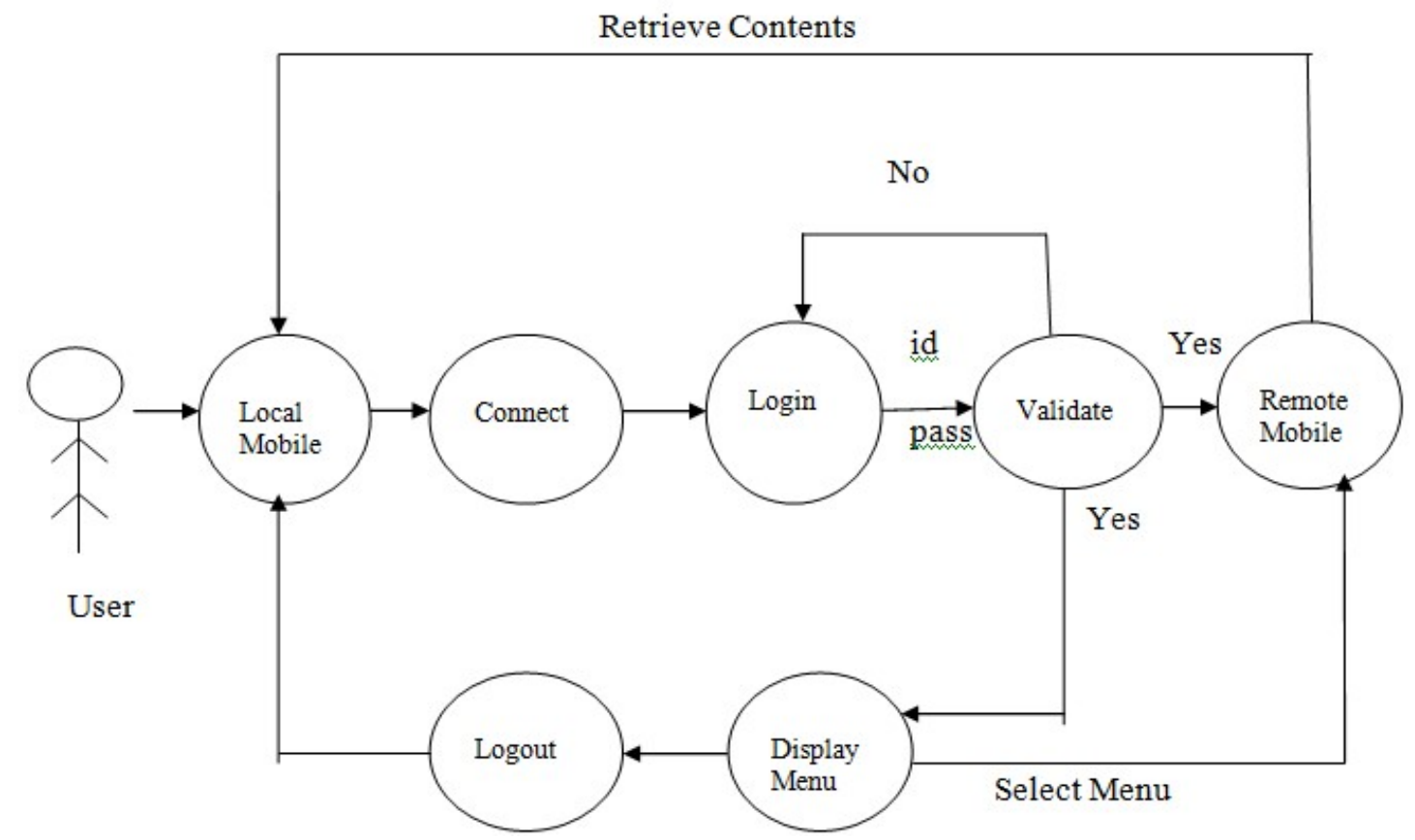

Fig. 2. Data Flow Diagram 
The figure shows the system data flow. It shows the user first take any other random mobile i.e. local mobile. User first send message for connection, so for user authentication there is login id and password, if user is not authentic then it returns to login stage to get correct user-id and password. If is correct the connection is established with random mobile. After login user can request to remote mobile to fetch some data, then it displays 'display menu' for ex. Contacts. The remote mobile respond as per request.

\section{B. UML DIAGRAMS}

A UML diagram is the pictorial representation of a elements, it state as a connected graph of vertices and arcs.

\section{Use Case Diagram [6]}

[2] Use case diagram shows requirements of system. These requirements are design requirements.

Here the user can send a message to remote mobile for connectivity, where it checks user validation, if validation is correct then through display menu user can send request to retrieve some data from remote mobile.

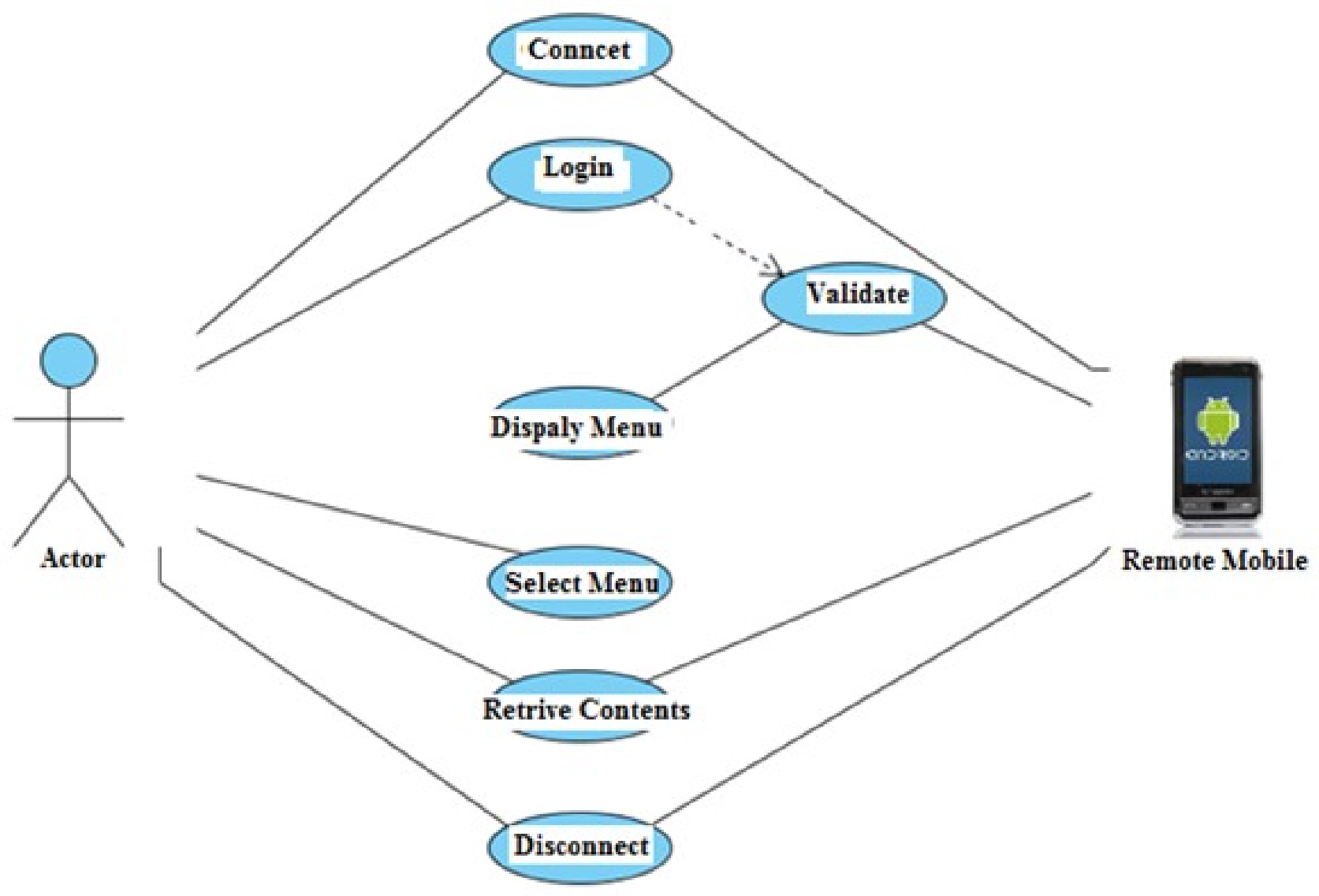

Fig. 3. Use Case Diagram

\section{Sequence Diagram}

Sequence diagram is one which shows how messages transfer between classes. Figure 4 shows the sequence diagram which shows classes User, Connection, Remote mobile. User send message to remote mobile to establish connection by login details. If login successful through display menu user can select menu and user can send message to remote mobile to fetch content. Remote mobile search requested content and send response. 


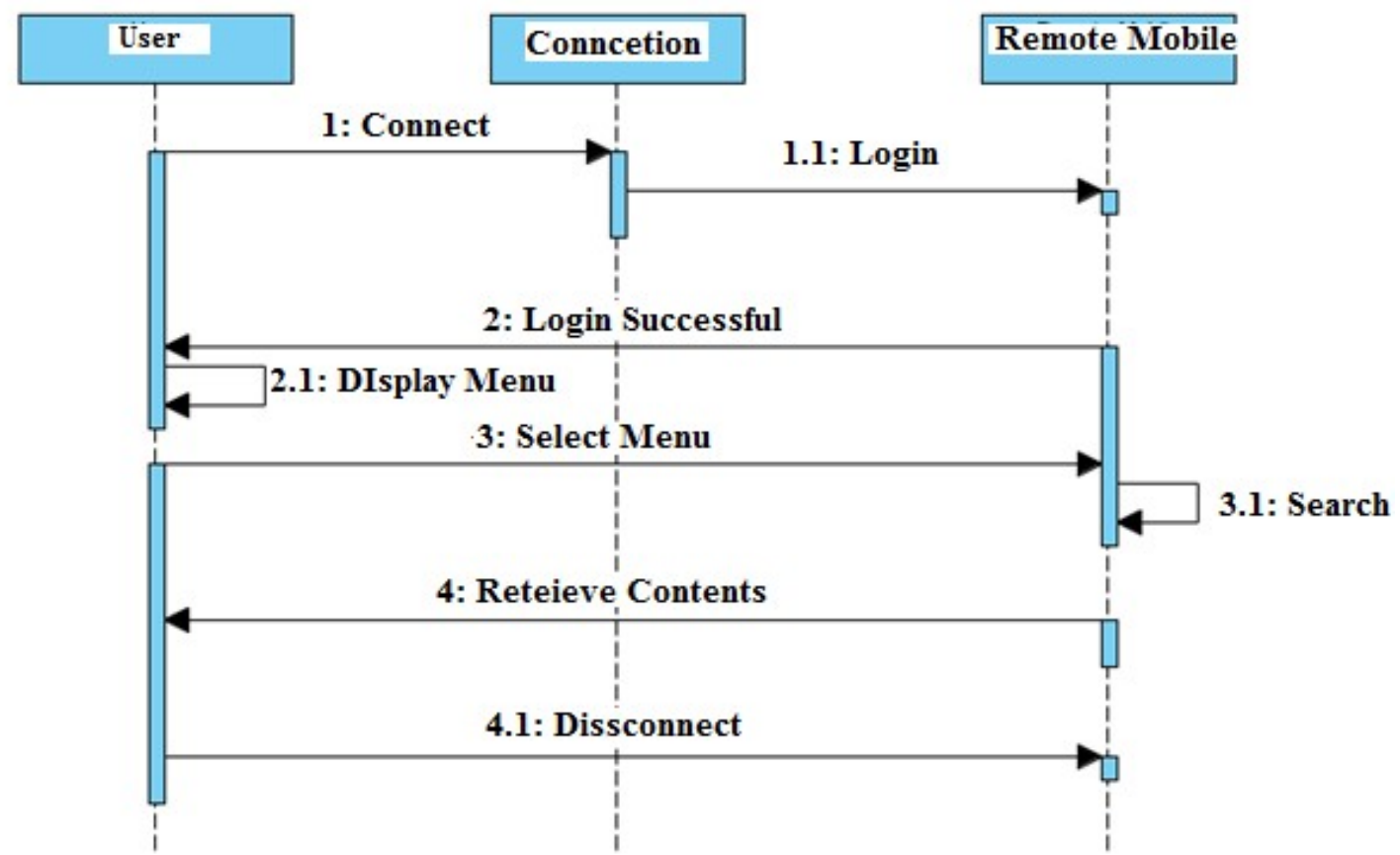

Fig. 4. Sequence Diagram

\section{State Diagram}

In the first step the connection is established. If the connection is established flow goes to next state where user will provide username and password. The provided username and password will be sent to remote phone for validation. Once user is authenticated he will see the menu on screen from which he has to select the menu item and send to the remote mobile. Then goes in state retrieve data from remote mobile.

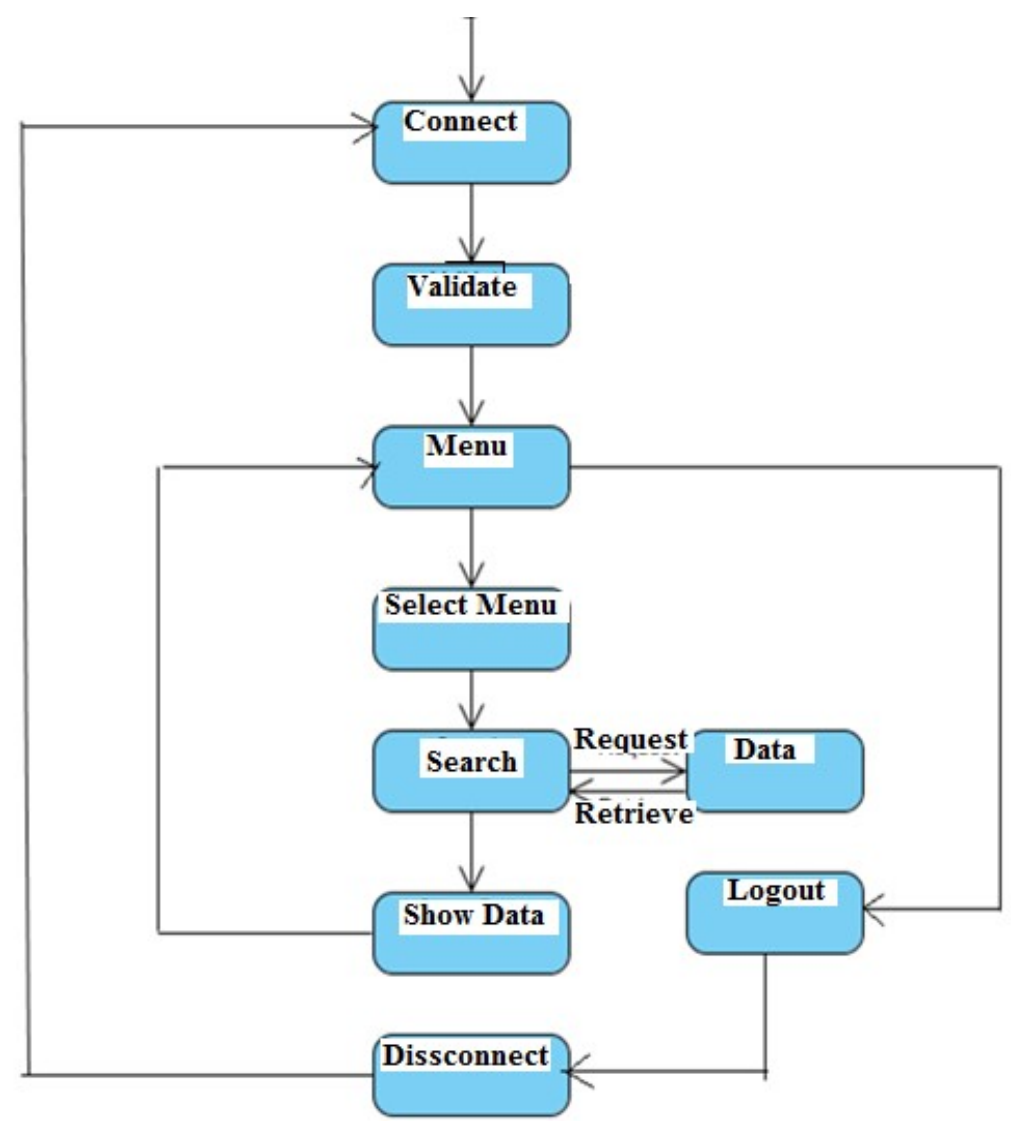

Fig. 5. State Diagram 
V. RESULTS

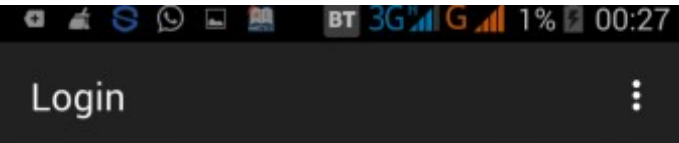

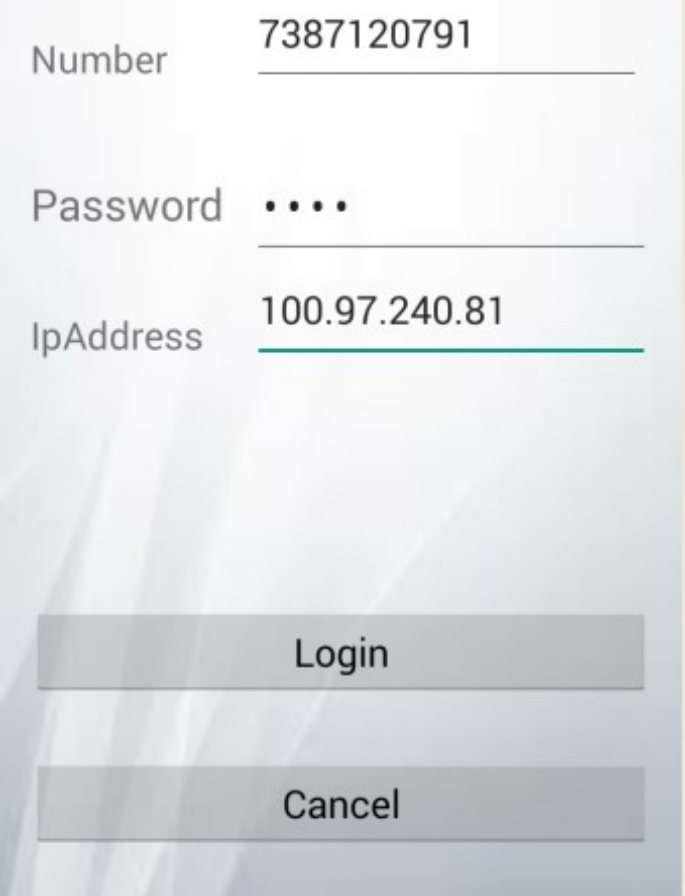

Fig. 6. Screenshot of Login

Figure 6 shows the login screen, this login screen mainly consists of inputs that user has to give for login .Here the mobile number as username and password for authentication and IP address to establish connection between two mobiles. The user has to give the same mobile number and password as he had given during installation of application. After feeling all the required inputs now user has to click on the "Login" button in order to traverse to the next screen. When the user click on login button the IP address of that mobile automatically binds to the edit text of Ip Address and one specific format of SMS will be sent to the mobile which is at home which will cost 1 Rs. After receiving the SMS, the application automatically starts on the mobile which is at home i.e. remote mobile. Then it tokenize the SMS and authenticate the SMS contents with the file contents which we have taken during installation. When authentication is successful it automatically turn on the data connection if it is off and connection is established using IP address in SMS. Once the connection is established it sends a message as "CONNECTED" to the local mobile.

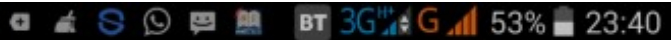

\section{ServerSocketNew}

\begin{tabular}{|c|}
\hline Contacts \\
\hline Files \\
\hline
\end{tabular}

app-7387120791-abcd-192.168.43.1

Fig. 7. Screenshot of Setting IP Address

Figure 7 shows ServerSocketNew screenshot, this screen will be displayed after receiving the message "CONNECTED". Here user has to select either CONTACTS or FILES. When user selects on CONTACTS screenshot, it displays next screen.

\section{(9)}

\section{ServerSocketNew}

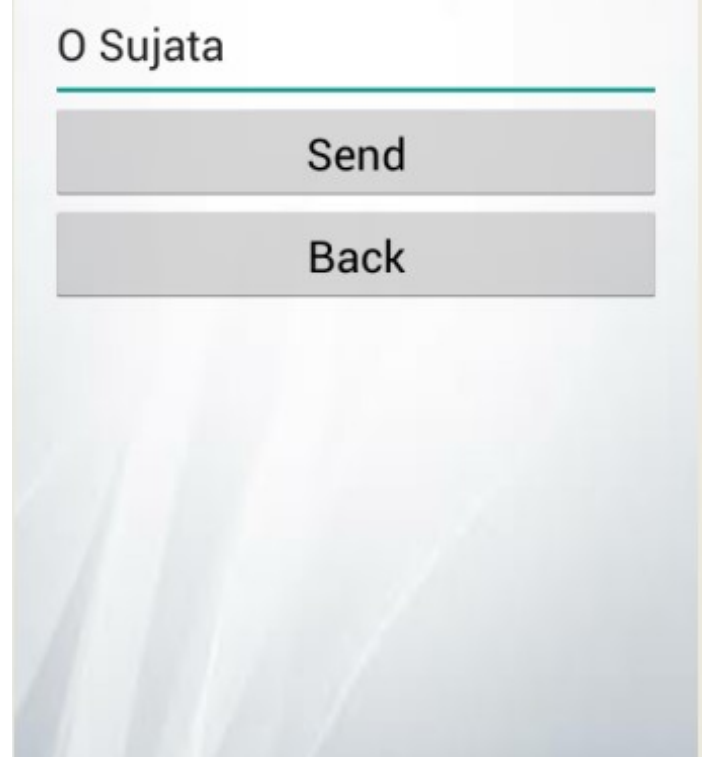

Fig. 8. Screenshot of Accessing Contact 
Figure 8 shows the third screen of the application. This screen will be displayed when user click on CONTACTS. Here user has to give the name of the person whose number he wants. After clicking on "send" button this name is send to the remote mobile which is at home. After few seconds, name with contact number is display in the list view.

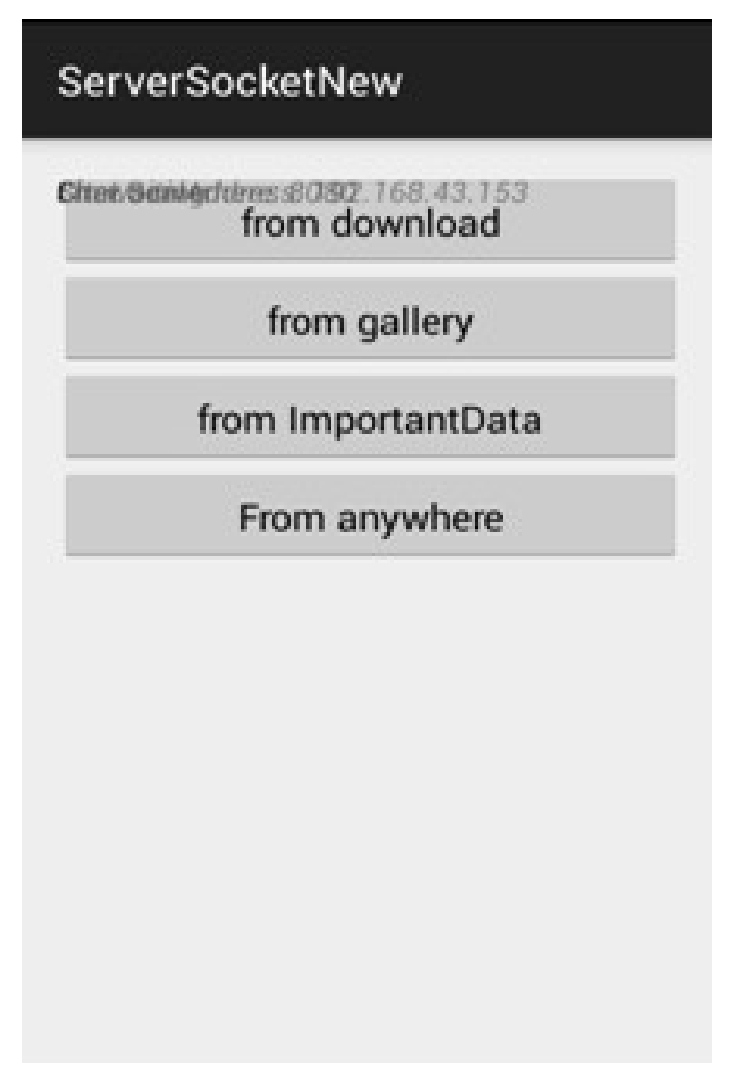

Fig. 9. Screenshot for Different Categories

Figure 9 shows the fourth screen of the application. This screen will be displayed when user click on FILES. This screen displays the four types of buttons name as From Download, From Gallery, From ImportantData and From Anywhere from where user can access the files. When user clicks on From Download then user gets all the file names from download folder of remote mobile. All the file names are display in the list view. From the list view user has to select the name of file. This procedure is same for From Gallery and From Important Data buttons. When user click on From Anywhere button then user has to know the entire path of file which he wants.

\section{CONCLUSION}

Now-a-days most of the people are doing their work using smart phones, because smart phones are providing all facilities of PC. The problem arises when the user forgets phone at home, and they want some data urgently from their phone. Hence the proposed system will come into picture where the user will have an application where he can retrieve data from his phone using any other smart phone. In this application user will have unique id and password for accessing their phone.
As almost every other person uses a smart phone so here is a really smart application which a user can take along with him to any place and can get the data urgently.

\section{REFERENCES}

[1] www.newkerala.com

[2] www.idt.mdh.se

[3] Rashmi A. Kalje, S. P. Kosbatwar , "Android Based Remote Control of Mobile Devices Using VNC System", International Journal of Computer Science and Information Technologies June 2012, Vol. 5.

[4] Abhishek Barve, Pragnesh shah, "Android based Remote Monitoring System", International Conference in Recent Trends in Information Technology and Computer Science 2012.

[5] P.L.Ramteke, D.N.Choudhary, "Smart Phone Access, Control \& Security", Advanced Engineering Website: International Journal of Emerging Technology, ISSN 2250-2459, Volume 2, Issue 6, June 2012

[6] Android Chinar Regundwar, Navnath Rahinj, Priti Rayrikar, Shashikant Navnath D. Kale, "88 Call Log, Message and Camera Monitoring System over Android", International Journal of Engineering and Advanced Technology, ISSN: 2249 - 8958, Volume-2, Issue-4, April 2013 